\title{
BSM searches in ATLAS
}

\section{Antonio Policicchio* \\ INFN LNF-CS}

E-mail: antonio.policicchiodcern.ch

Results are presented for searches beyond the Standard Model (SM) at the LHC by the ATLAS collaboration. The minimal supersymmetric extension of the SM has been investigated in various configurations at $8 \mathrm{TeV}$ centre of mass energy at the LHC and lower limits are set on the sparticle masses. Searches for other physics scenarios beyond the SM are also presented. The data are found to be consistent with the Standard Model. The non-observation of a signal is converted to limits at the $95 \%$ confidence level (CL) on the production cross section times branching ratio and/or on the masses of the new particles for appropriate benchmark models.

Proceedings of the Corfu Summer Institute 2014 "School and Workshops on Elementary Particle Physics and Gravity",

3-21 September 2014

Corfu, Greece

\footnotetext{
${ }^{*}$ Speaker.

$\dagger$ On behalf of the ATLAS Collaboration
} 


\section{Introduction}

While the Standard Model (SM) is very successful in describing most of the phenomena that we can experimentally investigate with the current generation of particle accelerators, it is generally believed to be only an effective theory that must break down above a certain energy scale, and there are strong theoretical arguments to believe that it breaks down at the electroweak scale. Some unexplained phenomena and problems, for example the Dark Matter, the difficulty of incorporating gravity and the hierarchy problem suggests that physics beyond the Standard Model (BSM) is required to accurately describe the universe we observe. Supersymmetry is proposed as a simple and elegant extension of the SM. Other theoretical approaches providing solutions to some of the problems mentioned above, and often offering a rich phenomenology, are grouped under the term of exotic physics. The ATLAS experiment [1] at the Large Hadron Collider (LHC) has run extensive physics searches to cover as many scenarios as possible. These proceedings review a selection of results among the many searches for supersymmetry and exotic signatures based on of protonproton collision data collected in 2012 at the LHC at a centre of mass energy of $\sqrt{s}=8 \mathrm{TeV}$.

\section{Exotics searches}

\subsection{Search for high-mass dilepton resonances}

The dilepton final state is generally one of the first to be studied as it benefits from a clear signature and a relatively low background. The main challenge is the transverse momentum resolution of the leptons. In the case where the two leptons are electrons or muons, ATLAS [2] selects two well isolated leptons. In the electron case, no charge identification is required. The main backgrounds originate from Drell-Yan, diboson, top and jet productions. Misidentified jets are an important background for the electron final states for which the fake rate is measured with data. The dilepton invariant mass distributions are shown in Figure 1. A good agreement between data and SM model predictions is found in all channels. Thus ATLAS sets limits on a Sequential Standard Model (SSM) $Z^{\prime}[3]$ as well as on a six extra space-time dimension $E_{6}, Z_{\Psi}$ or $Z_{\chi}$ [4, 5]. Results are also interpreted in terms of a $Z^{*}$ from a $\left(Z^{*}, W^{*}\right)$ doublet, and a spin 2 Randall-Sundrum (RS) graviton excitation $\left(G^{*}\right)$ [6]. The limits are in the 1.0 to $2.9 \mathrm{TeV}$ range and a SSM $Z^{\prime}$ is excluded below $2.90 \mathrm{TeV}$. Exclusion limits are shown in Figure 2.

\subsection{Search for vector diboson resonances: $W Z, W W, Z Z$}

Diboson analyses have been performed in all the possible final states [8, 9]. The studies reported here are for the lepton plus jets and fully leptonic final states. In the lepton plus jet channel, ATLAS looks for two small radius jets (radius parameter $\left.R_{j e t}=0.4\right)$ or one big jet $\left(R_{\text {jet }}=\right.$ 1.2). The splitting allows for a good sensitivity at low mass. ATLAS sets a limit on an Extended Gauge Model (EGM) $W^{\prime}[3]$ at $1.59 \mathrm{TeV}$ and on a RS bulk $G^{*}$ at $740 \mathrm{GeV}$ for a coupling parameter value of $k / M_{P I}=1.0$ [6]. The fully leptonic channel is used to search for a new resonance decaying to $W Z$. It shows a better sensitivity at low mass thanks to its higher mass resolution and its smaller backgrounds that come mainly from SM diboson production as well as top-antitop production associated with a $W$ or a $Z$. The main uncertainties are those associated with the PDFs and the 

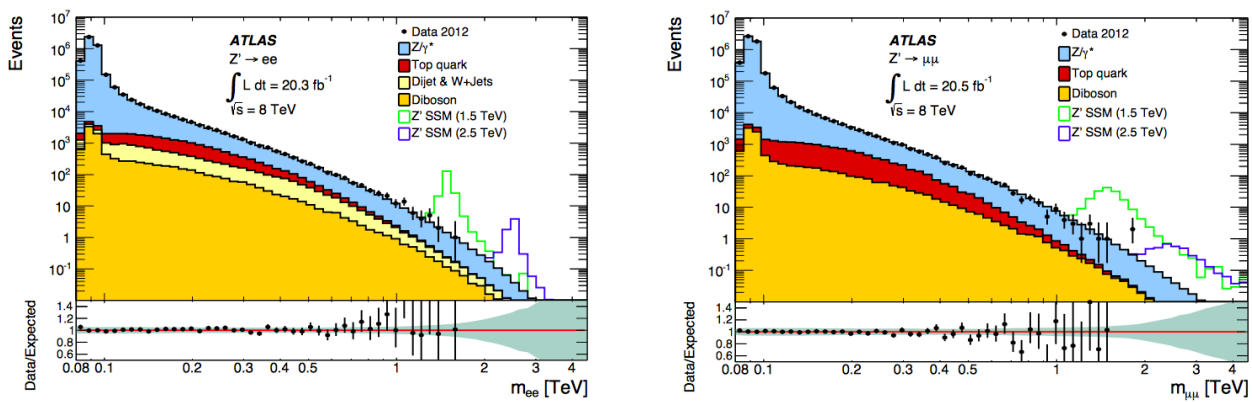

Figure 1: Dielectron (left) and dimuon invariant mass distributions (right), with two selected SSM $Z^{\prime}$ signals overlaid, compared to the stacked sum of all expected backgrounds, and the ratios of data to background expectation. The green band in the ratio plot shows the systematic uncertainties [2].

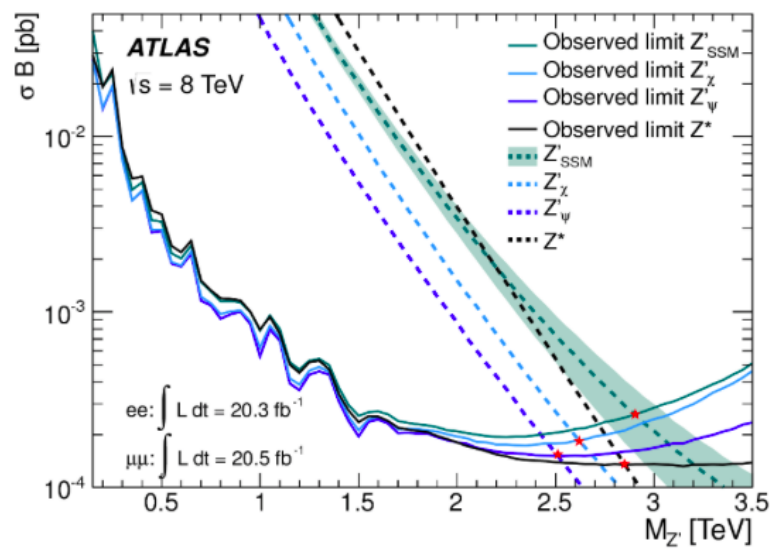

Figure 2: Observed cross-section times branching ratio upper limits for various models in the dilepton final state [2].

normalisation and factorisation scales for the backgrounds. A good agreement is found with the SM prediction leading to the exclusion of an EGM $Z^{\prime}$ below $1.5 \mathrm{TeV}$, as shown in Figure 3.

\subsection{Resonant Higgs pair production}

ATLAS has searched for the first $K K$ excitation of the bulk RS graviton $G^{*}$ [6] that decays into two Higgs where each boson decays to a $b$ quark pair [10]. The main backgrounds are multijet and top pair production. Dijet that are b-tagged and near the Higgs mass are selected and a top-pair veto is used. The multijet background is estimated using data. A good agreement is found with SM expectations and a graviton mass between 590 and $710 \mathrm{GeV}$ is ruled out for $k / M_{P I}=1.0$.

\subsection{Dijet mass resonances}

The dijet final state is challenging since the massive object that couples to quarks and gluons has to be found in the huge multijet QCD background. The ATLAS search [11] uses a single jet pre-scaled trigger to be sensitive at low mass. The analysis relies on data to evaluate the background. The main uncertainties are those associated with the jet energy scale and resolution and the luminosity measurement. Results are given in a model independent way. In addition, this anal- 


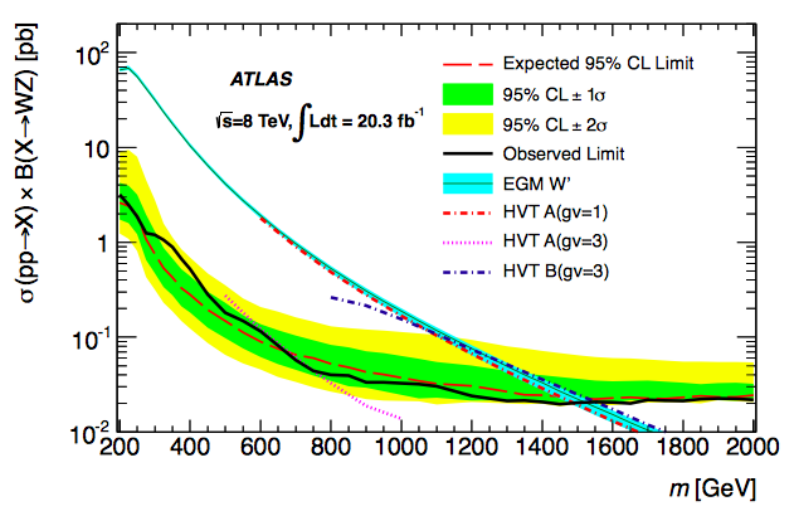

Figure 3: Observed cross-section times branching ratio upper limits as a function of the signal mass $\mathrm{m}$, where $\mathrm{X}$ stands for the signal resonance. The expected limits are also shown together with the \pm 1 and \pm 2 standard deviation uncertainty bands. Theoretical cross sections for the EGM $W^{\prime}$ and the Heavy Vector Triplet (HVT) [7] benchmark models are also shown. The uncertainty band around the EGM $W^{\prime}$ crosssection line represents the theoretical uncertainty on the NNLO cross-section calculation using ZWPROD [8].

ysis excludes excited quarks [12] with a mass below $4.06 \mathrm{TeV}$, heavy vector bosons [13] with a mass below $2.45 \mathrm{TeV}$, chiral bosons [14] with a mass below $1.75 \mathrm{TeV}$, and quantum black holes with six extra space-time dimensions [15] with threshold mass below $5.66 \mathrm{TeV}$.

\subsection{Microscopic black holes}

Models with additional space dimensions which are compactified such that they are not part of our daily perception can explain the huge difference of the strengths of electroweak and gravitational interactions. A consequence of these models is the possible production of microscopic black holes. Once these are produced they evaporate immediately through Hawking radiation and leave a distinct experimental signature in the experiment. Here an analysis is discussed [16] which selects events with at least three objects (leptons or jets) with transverse momenta of at least $100 \mathrm{GeV}$. The sum of the total transverse momentum in the event is then used to search for the spectacular signature of a microscopic black hole. Standard Model backgrounds which have been determined using MC simulations normalized to data using appropriate control regions, predict a steeply falling distribution whereas models with black holes show an enhancement of events with large values of the total transverse energy. The absence of a deviation from the predicted steeply falling spectrum can be translated into limits on the production threshold for black holes on the order of 5 to $6 \mathrm{TeV}$. The exact values depend on additional details of the particular theories.

\subsection{Non-prompt lepton jets}

Several models of physics beyond the Standard Model predict neutral particles (dark-photons, $\gamma_{d}$ ) that decay into final states consisting of collimated jets of light leptons and hadrons (so-called "lepton jets"). These particles can also be long-lived with decay length comparable to, or even larger than, the LHC detectors' linear dimensions. ATLAS has searched for non-prompt lepton jets in all the possible final states: only muons, only electrons, mixed electrons-muons and mixed electrons/muons-light hadrons [17]. The high-resolution, high-granularity measurement capability 
of the ATLAS Muon Spectrometer is crucial for this type of search. In addition, the ATLAS Inner Tracker can be used to define isolation criteria to significantly reduce, for decay vertices far from the interaction point, the otherwise overwhelming SM background from proton-proton collisions. No excess of events has been observed over the estimated background level, and limits have been set on the Falkowski-Ruderman-Volansky-Zupan (FRVZ) models [18] which predict non-SM Higgs boson decays to lepton jets. The limits are set on the Higgs boson decay branching fraction to lepton jets as a function of the dark photon mean lifetime. In the case of a dark photon which kinetically mixes with the SM photon, these limits can be converted into exclusion limits on the kinetic mixing parameter $\varepsilon$. For $H \rightarrow 2 \gamma_{d}+X$ with a dark photon mass $=0.4 \mathrm{GeV}$, the interval that is excluded at $95 \% \mathrm{CL}$ is $7.7 \times 10^{-7} \leq \varepsilon \leq 2.7 \times 10^{-6}$. The final results of the search and their contribution to the parameter space exclusion plot for dark photons are shown in Figure 4.

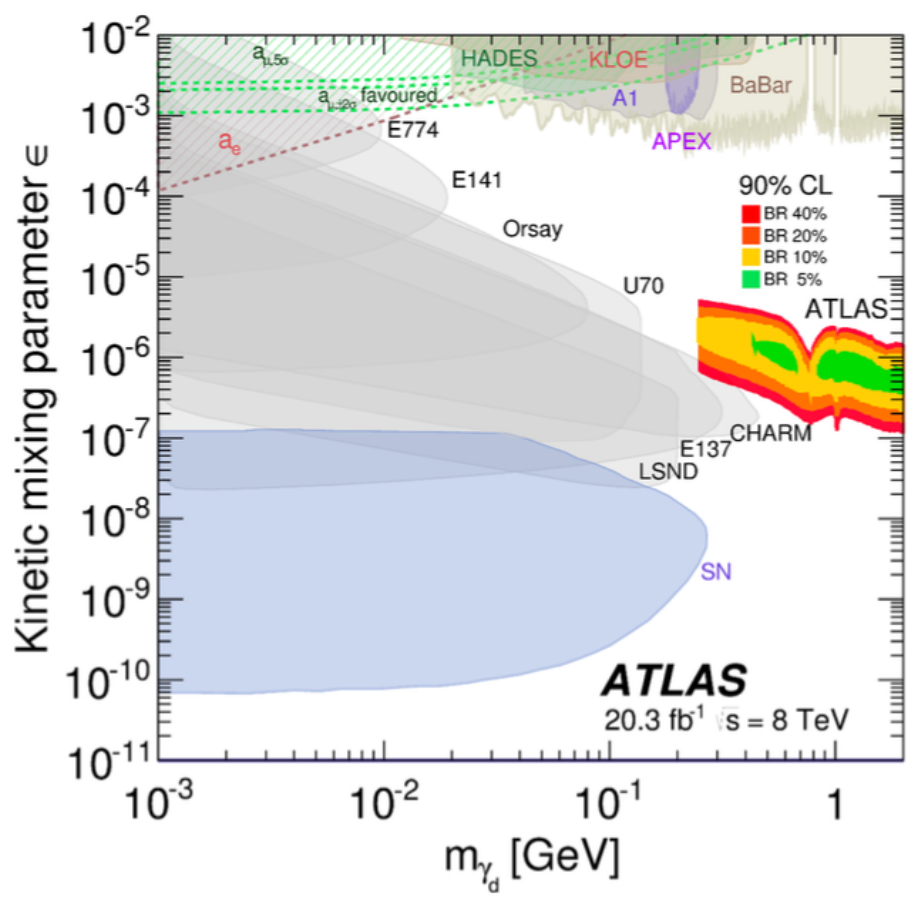

Figure 4: Parameter space exclusion plot for dark photons as a function of the dark photon mass and of the kinetic mixing parameter [17]. Shown are existing 90\% CL exclusion regions from beam dump experiments E137, E141, and E774, Orsay, U70, CHARM, LSND, A1, the electron and muon anomalous magnetic moment, HADES, KLOE, the test run results reported by APEX, an estimate using a BaBar result, and constraints from astrophysical observations. The $90 \% \mathrm{CL}$ exclusion limits from the ATLAS search, assuming the FRVZ model $H \rightarrow 2 \gamma_{d}+X$ with decay branching fraction to dark photon of 5/10/20/40\% and the NNLO gluon fusion Higgs production cross section, are shown.

\section{Searches for Supersymmetry}

Analyses searching for Supersymmetry (SUSY) typically involve identifying some signature of interest expected from a SUSY model. Simplified models are often used to represent a signal, where only a few SUSY particles have light enough masses to be produced at the LHC, and the 
physics above this scale, i.e. the SUSY breaking mechanism, is not considered. Once a signature has been identified, the objective is to isolate event or object properties where we would expect good discrimination between the SM background and our signal. This is usually done in the form of a set of kinematic selections or cuts required for events to pass the requirements of a signal region, and all SUSY searches discussed in this document are based on this method. If agreement is seen between the SM prediction and the observed data, 95\% CL exclusion limits are set, ruling out scenarios that are incompatible with observations. These limits are usually given as a function of parameters in the model. In order to interpret these observations effectively, it is vital that the SM background processes are well modelled and accurately described in the regions of interest. Analyses often incorporate data-driven background estimation methods where possible, and validate the predictions in specifically designed control regions. Many SUSY searches are conducted blind. This means that the signal regions are designed based solely on simulation, and the data are not examined until everything in the analysis is finalized. This is referred to as unblinding. For these analyses it is crucial that this testing of the background estimation does not uncover data in the search regions prior to unblinding, so validation of the background estimation must be done in regions that do not have any overlap with the signal regions.

\subsection{Strong production searches}

Direct production of gluinos and first and second generation squarks have high cross-sections for masses up to around $1 \mathrm{TeV}$. The majority of models consider the decay of these SUSY particles to the lightest sparticle (LSP), which is usually considered to be a neutralino. This will escape the experiment undetected, provided it is stable. In these cases, the LSP provides a good candidate for a weakly interacting dark matter particle. The decay chain leading from the strongly produced sparticles to the LSP will involve the production of a number of SM particles, predominantly hadronic jets and, depending on the scenario, sometimes leptons. The escaping LSP provides a source of missing transverse energy, a requirement on which is also commonly included in searches. Results of the ATLAS searches [19, 20] are summarized in Figure 5, which shows the exclusion limit for gluino production, in the case of a neutralino LSP. Different analyses (listed in the legend) are useful for different areas of the parameter space, and the maximum effect is gained by considering all final states with sufficient sensitivity.

\subsection{Third generation searches}

Direct production of the third generation strongly-interacting sparticles, i.e. the stops and sbottoms, are considered separately from other SUSY ATLAS searches [23, 24]. They have a more moderate production cross-section than the gluinos and first and second generation squarks, up to masses of around $0.5 \mathrm{TeV}$. The decays of these particles also produce a large number of jets, possibly leptons, and missing transverse energy. Figure 6 summarises ATLAS results from a number of analyses in the $\tilde{\chi}_{1}^{0}$ mass versus $\tilde{t}_{1}$ mass parameter space. Different regions of this space are accessed using different decay modes of the stop, as these are dependent on the mass different between the $\tilde{t}_{1}$ and $\tilde{\chi}_{1}^{0}$. Lines are included to mark the limits of a given decay mode, as labelled. The analysis contributes to three regions of the parameter space via these different decay modes, as can be seen by the three distinct exclusion regions displayed. 


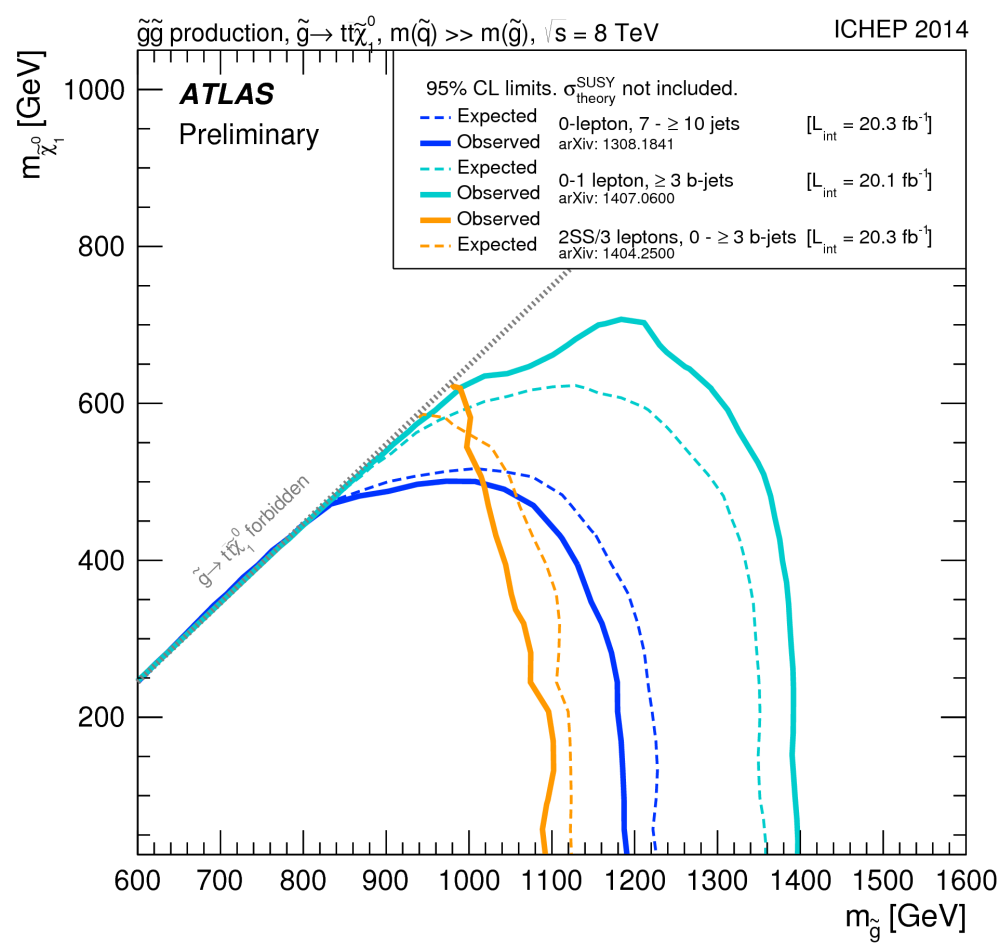

Figure 5: Exclusion limits at 95\% CL for $8 \mathrm{TeV}$ analyses in the $\tilde{\chi}_{1}^{0}$ mass versus $\tilde{g}$ mass plane for the Gtt simplified model [21] where a pair of gluinos decays promptly via off-shell stop to four top quarks and two lightest neutralinos (LSP) [22]. Theoretical signal cross section uncertainties are not included in the limits shown.

\subsection{Electroweak production}

In the limit that the strongly produced sparticles are most often produced off-shell, production of the electroweakinos may be the dominant SUSY process at the LHC, and will most likely be found via multilepton final states. Direct production of charginos $\left(\tilde{\chi}^{ \pm}\right)$and neutralinos $\left(\tilde{\chi}_{i}^{0}\right)$, considering the $\tilde{\chi}_{1}^{0}$ as LSP, is expected to have a clean signature due to the presence of SM bosons and leptons in the decay chains. Depending on the mass splitting, a heavier neutralino can decay to the LSP via a $Z$ or the SM Higgs, and the charginos via a $W$. The final states in direct pair production of charginos and neutralinos are characterised by a large lepton multiplicity $(\geq 2)$. Depending on the decay, a dilepton invariant mass compatible with the $Z$ mass can be vetoed or required. Chargino masses below $350 \mathrm{GeV}$ are excluded for the decay via SM bosons and $700 \mathrm{GeV}$ for the decays via sleptons [25]. The summary of all ATLAS electroweakinos searches can be seen in Figure 7.

\subsection{R-parity violating searches}

R-parity violating supersymmetry scenarios can lead to long-lived particles (LLPs) if the coupling is weak enough, giving a displaced decay of the metastable LSP. The ATLAS long-lived slepton search [26] for charged LLPs with $c \tau>1 \mathrm{~m}$ has results interpreted in the context of a $\tilde{\tau}_{1}$ in models with Gauge Mediated SUSY Breaking [27]. LLPs with $c \tau>1 \mathrm{~m}$ are likely to decay outside ATLAS and, if charged, leave a track similar to a muon throughout the detector systems. LLPs are differentiated from muons using track $\beta$ values calculated from $d E / d x$ measurements in 


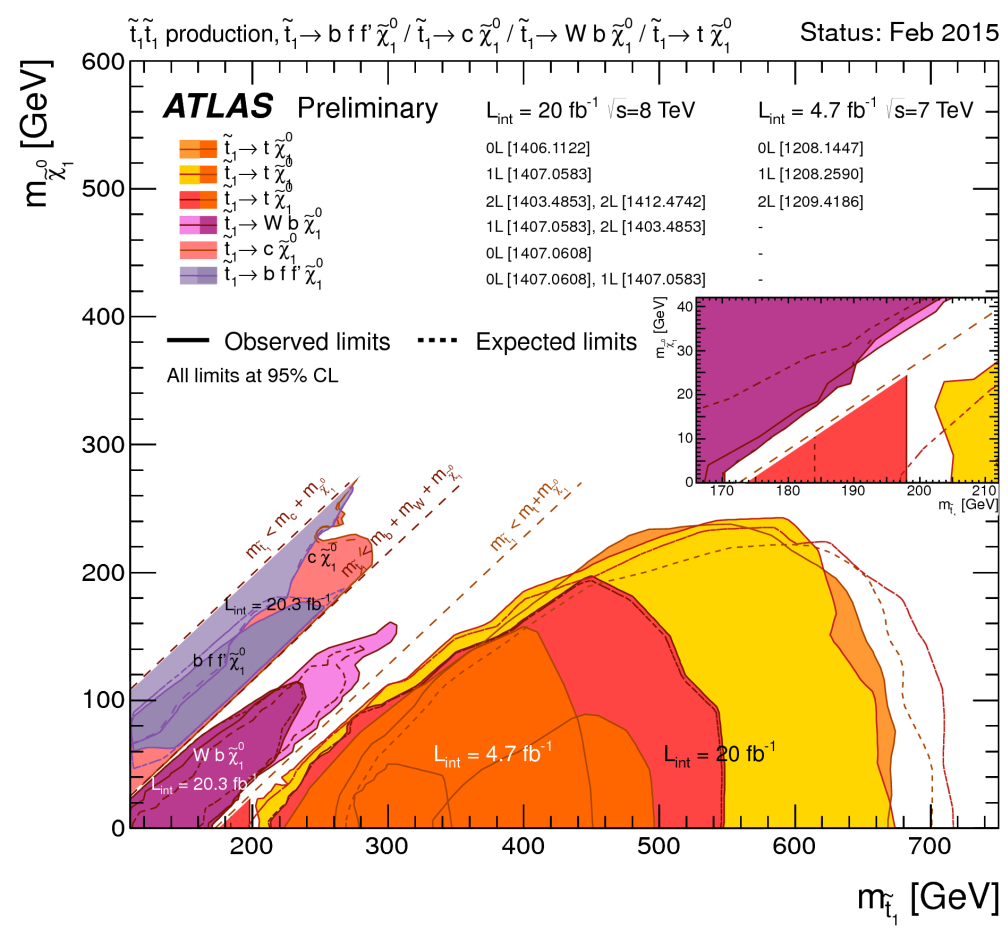

Figure 6: Summary of $95 \%$ CL exclusion limits in strong production scenarios in the $\tilde{\chi}_{1}^{0}$ mass versus $\tilde{t}_{1}$ mass plane [22].

the Inner Tracker pixel detectors and time-of-flight measurements in the Calorimeters and Muon Spectrometer. Tracks are considered to be LLP candidates if they have transverse momentum $>50$ $\mathrm{GeV}$ and $\beta<0.95$. The background to this analysis is overwhelmingly high transverse momentum muons with mismeasured $\beta$ values. Background is reduced by requiring two LLP candidates in an event and the signal region is set by calculating candidate masses from their transverse momentum and $\beta$, before applying a model dependant mass cut. For the model parameter range $\tan \beta=5-50$, limits excluding $m_{\tilde{\tau}_{1}}<347-402 \mathrm{GeV}$ can be set.

The stopped R-Hadron analysis [28] considers the possibility of R-hadrons that lose all kinetic energy, stopping inside the calorimeter and then decaying outside of the event timing window. The search is performed by looking for jets in "empty" bunch crossings (no protons in either beam). Background from cosmic rays is effectively suppressed by vetoing events with reconstructed muon segments. Excluding events with spike-like signals in the calorimeter rejects background from random electronic noise and also reduces the effect of cosmic rays and beam halo. The analysis requires events with an $E_{T}>50 \mathrm{GeV}$ jet and no more than six jets in total; a requirement that the event transverse missing energy be more than half of the leading transverse momentum jet is also applied. Depending on model parameters, limits excluding $m_{\tilde{g}}<545-832 \mathrm{GeV}$ and $m_{\tilde{q}}<344-397$ $\mathrm{GeV}$ can be set.

\section{Conclusions}

ATLAS is conducting a wide range of searches for SUSY particles and other exotic new particles, exploiting different signatures and using various detection techniques. These proceedings 


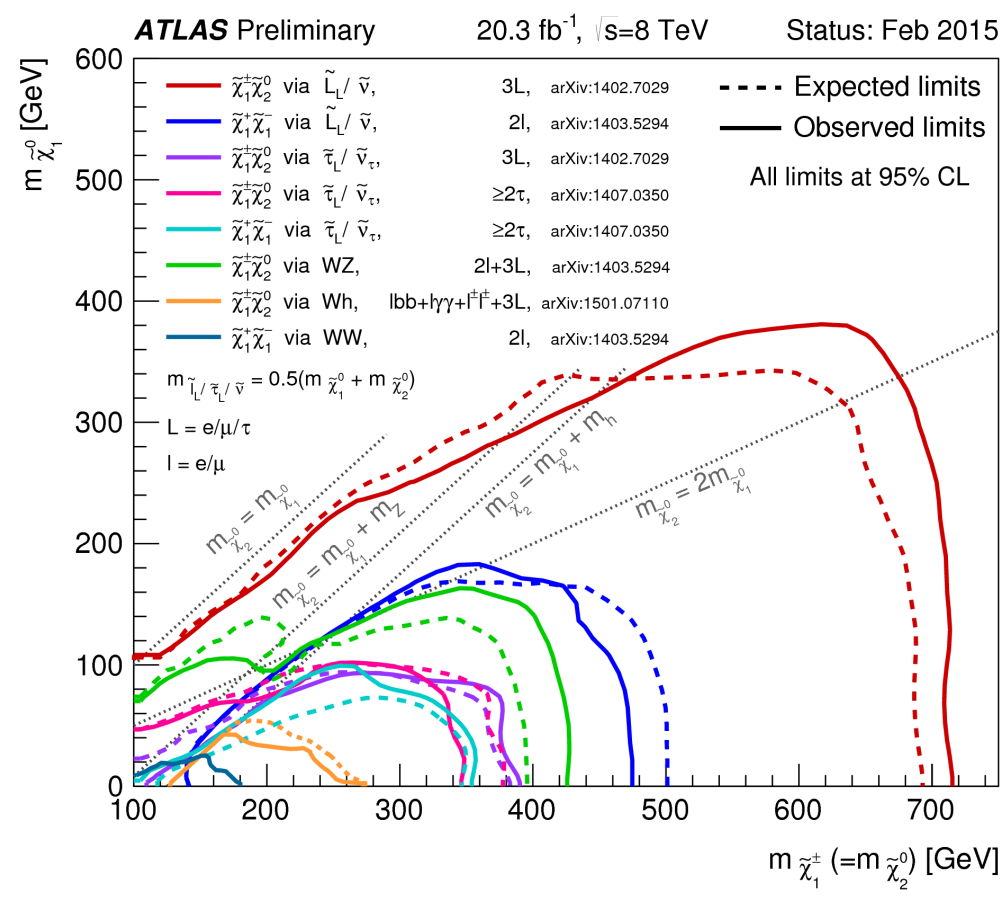

Figure 7: Summary of ATLAS search for electroweak production of charginos and neutralinos. Limits are set in the chargino/neutralino mass planes. The dashed and solid lines show the expected and observed limits, respectively, including all uncertainties except the theoretical signal cross section uncertainties [22].

give a summary of the result of some of these searches. Despite a huge effort, no exotic signal has been observed in the data to date. Thus, exclusion limits on cross sections and masses are given for various models.

\section{References}

[1] The ATLAS Collaboration, JINST 3 (2008) S08003.

[2] The ATLAS Collaboration, Phys. Rev. D. 90, 052005 (2014).

[3] Altarelli G, Mele B and Ruiz-Altaba M, Z. Phys. C 45 (1989) 109.

[4] D. London and J. L. Rosner, Phys. Rev. D34 (1986) 1530.

[5] P. Langacker, Rev. Mod. Phys. 81 (2009) 1199.

[6] L. Randall and R. Sundrum, Phys. Rev. Lett. 83 (1999) 3370.

[7] D. Pappadopulo, A. Thamm, R. Torre, A. Wulzer, arXiv:1402.4431 (2014).

[8] The ATLAS Collaboration, Phys. Lett. B 737 (2014), 223-243.

[9] The ATLAS Collaboration, Eur. Phys. J. C (2015) 75:69.

[10] The ATLAS Collaboration, Phys. Rev. Lett. 114, 081802 (2015).

[11] The ATLAS Collaboration, Phys. Rev. D. 91052007 (2015).

[12] U. Baur, M. Spira, and P. M. Zerwas, Phys. Rev. D 42, 815 (1990). 
[13] H. Georgi, E. E. Jenkins, and E. H. Simmons, Nucl. Phys. B 331, 541 (1990).

[14] M. Chizhov, Phys. Part. Nucl. Lett. 8, 512 (2011).

[15] L. A. Anchordoqui, J. L. Feng, H. Goldberg, Shapere, Phys. Lett. B 594, 363 (2004).

[16] The ATLAS Collaboration, JHEP 08 (2014) 103.

[17] The ATLAS Collaboration, JHEP 11 (2014) 088.

[18] A. Falkowski, J.T. Ruderman, T. Volansky and J. Zupan, JHEP 05 (2010) 077.

[19] The ATLAS Collaboration, JHEP 09 (2014) 176.

[20] The ATLAS Collaboration, JHEP 10 (2014) 024.

[21] The ATLAS Collaboration, Eur.Phys.J. C72 (2012) 2174.

[22] The ATLAS Collaboration, https://twiki.cern.ch/twiki/bin/view/AtlasPublic/SupersymmetryPublicResults.

[23] The ATLAS Collaboration, JHEP 09 (2014) 015.

[24] The ATLAS Collaboration,t JHEP 11 (2014) 118.

[25] The ATLAS Collaboration, JHEP 04 (2014) 169.

[26] The ATLAS Collaboration, JHEP 01 (2015) 068.

[27] M. Dine and W. Fischler, Phys. Lett. B 110 (1982) 227.

[28] The ATLAS Collaboration, Phys. Rev. D 88 (2013) 112003. 